\title{
High Prevalence of 16S rRNA methylase genes High Prevalence of 16S rRNA methylase genes Among Carbapenem-resistant Hypervirulent Klebsiella pneumoniae Isolates in China
}

\author{
Wenjian Liao ( $\nabla 97854867 @ q q . c o m$ )

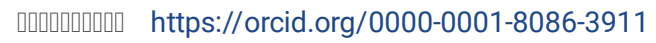 \\ Dan Li \\ First Affiliated Hospital of Nanchang University \\ Dan Dan Wei \\ First Affiliated Hospital of Nanchang University \\ Fang-lin Du \\ First Affiliated Hospital of Nanchang University \\ Dan Long \\ First Affiliated Hospital of Nanchang University \\ Wei Zhang \\ First Affiliated Hospital of Nanchang University \\ Yang Liu \\ First Affiliated Hospital of Nanchang University
}

Research article

Keywords: plasmid-mediated 16S rRNA methylase, carbapenem-resistant, K.pneumoniae, hypervirulence

Posted Date: September 23rd, 2019

DOI: https://doi.org/10.21203/rs.2.14808/v1

License: (c) (i) This work is licensed under a Creative Commons Attribution 4.0 International License. Read Full License 


\section{Abstract}

Background : the existence of $16 \mathrm{~S}$ rRNA methylase genes would increase treatment difficulty of patients infected with CR-hvKP strains, this study was aimed to testify the prevalence of the 16S rRNA methylase genes genes in the CR-hvKP strains in China.Methods : Thirty-nine carbapenem-resistant hypervirulent Klebsiella pneumoniae (CR-hvKP) isolates collected from a Chinese hospital during the whole year of 2018 were evaluated to characterize the prevalence of $16 \mathrm{~S}$ rRNA methylase genes. Results : In tatal $66.7 \%(26 / 39)$ of the CR-hvKP isolates were found to carry $16 \mathrm{~S}$ rRNA methylase genes, and the most frequently detected gene was armA (11,42.3\%), followed by rmtB (8,30.8\%), and 7 CR-hvKP strains were found to carry both armA and rmtB (26.9\%). All the clinical isolates were found to carry at least one carbapenemase gene,with KPC-2 (79.5\%,31/39), NDM-1 (10.3\%,4/39), and cocarrying KPC-2 and NDM-1 (10.3\%,4/39). A total of 89.7\% (35/39) isolates carried ESBL genes, including 61.5\% (24/39) blaSHV-1 ,71.8\% (28/39) blaTEM-1 and 89.7\% (35/39) blaCTX-M-1 4. All except four isolates (89.7\%,35/39) harbored PMQR genes,with qnrS (82.1\%,32/39), aac(6')-Ib-cr (79.5\%,31/39), qnrB $(2.6 \%, 1 / 39)$.All the 16S rRNA methylase genes-positive CR-hvKP strains were firstly found to cocarry carbapenemase genes, ESBL genes and PMQR genes simultaneously. The most prevalent virulence genes were rmpA2 and entB (100\%, 39/39),followed by silS (97.4\%, 38/39), ybtS (94.9\%, 37/39), iutA $(92.3 \%, 36 / 39)$, kpn (92.3\%, 36/39), rmpA (87.2\%, 34/39), terW (84.6\%, 33/39), aerobactin (23.1\%, 9/39), repA (17.9\%, 7/39), magA $(10.3 \%, 4 / 39)$, kfuB C $(10.3 \%, 4 / 39)$, w ca G $(10.3 \%, 4 / 39)$, allS (10.3\%, 4/39). Multilocus sequence typing (MLST) analysis assigned the 39 CR-hvKP isolates into 4 sequence types (STs), with ST11 encompassing $79.5 \%$ of the strains. Pulsed field gel electrophoresis (PFGE) typing showed that strains closely related by MLST clustered in major PFGE clusters, of which cluster A accounts for 31 ST11 isolates. The analysis of the transconjugants showed a high-level aminoglycoside resistance and a popular cotransfer of bla KPC-2 with the 16S rRNA methylase genes.Conclusions : 16S rRNA methylase genes are highly prevalent in CR-hvKP clinical isolates especially for ST11, it is therefore critical to continuously monitor the 16S rRNA methylase-producing CR-hvKP epidemiology and minimize potential risks from aminoglycoside -resistant CR-hvKP.

\section{Background}

Klebsiella pneumoniae is one of the common pathogens of nosocomial infections including bloodstream infections, pneumonia, urinary tract infections and liver abscesses[1].Recently carbapenem-resistant hypervirulent klebsiella pneumoniae (CR-hvKP) infections have been reported widely in China[24].Due to acquisition of the carbapenem-resistance plasmid by the hvKP strains or the acquisition of the virulence plasmid by the CRKP strains, CR-hvKP strains simultaneously exhibit the features of hyper-resistance, hypervirulence, and high transmissibility so that they should be regarded as a real superbug which need to raise enough concerns[5].

In spite of ototoxicity and nephrotoxicity, aminoglycosides (AGs) are few optional semisynthetic antimicrobial agents exhibiting high-susceptibility and excellent post-antibiotic effect against CRKP strains[6,7].The biological mechanisms of resistance to aminoglycosides include decreased permeability, increased efflux, enzymatic modification, and modifications of the 30S ribosomal subunit that interferes with binding of the aminoglycosides[8]. Over the past few decades some studies have found alarmingly high rate of 16S rRNA methylase genes among CRKP strains[9,10], it precludes the use of key aminoglycosides (gentamicin, tobramycin, and amikacin) even when carbapenems have already been excluded from the treatment option. Obvious the existence of 16S rRNA methylase genes would increase treatment difficulty of patients infected with CR-hvKP strains. Thus, this study was aimed to testify the prevalence of the 16S rRNA methylase genes genes in the CR-hvKP strains in China.

\section{Methods}

\section{Bacterial strains and antimicrobial susceptibilities}

A total of 513 nonduplicate Klebsiella pneumoniae clinical isolates were collected from the First Affiliated Hospital of Nanchang University in the southeastern region of China from January to December 2018.Among these, 39 CR-hvKP clinical isolates were selected from clinical specimens, including 21 blood, 14 sputum, 1 pus, 1 urine, and 2 other specimen sources,respectively. According to the latest definition of hvKP[11], we selected the CRKP strains carrying the pLVPK-like virulence plasmid as CR-hvKP strains in this study. K.pneumoniae isolates were identified by an automated Vitek II system (bioMe'rieux,Balmes-les-Grottes,France) and were further verified with 16S rRNA gene sequencing. Antibiotic susceptibilities were determined by the disk diffusion method on Mueller-Hinton agar according to the Clinical and Laboratory Standards Institute (CLSI) guidelines[12].Escherichia coli ATCC 25922 and K. pneumoniae ATCC 700603 were used as the quality control. E. coli J53 was used in the conjugation experiments.

\section{PCR detection of resistance genes and virulence-associated genes}

The DNA was extracted with the boiling method in sterile distilled water for ten minutes.Single PCR was used to analyze genes PMQR ( $q n r A$, qnrB, qnrS, qepA, and $\left.\operatorname{aac}\left(6^{\prime}\right)-I b-c r\right)$ [13], ESBLs (CTX-M, SHV, TEM) [14], and carbapenemase genes (KPC, IMP, VIM, NDM, OXA-48) [15] with specific primers for each one, as previously reported.Additionally, all isolates were screened for the presence of the 16S rRNA methylase genes, including armA, $r m t A, r m t B, n p m A, r m t C, r m t D$, by PCR and DNA sequencing[16]. Capsular serotyping gene $K 1, K 2$ and fourteen virulence genes, including aerobactin, rmpA2, terW, kfuBC, rmpA, alls, silS, iutA, ybtS, wcaG, kpn, entB, magA, and repA, were identified using polymerase chain reaction (PCR) amplification as described in previous papers[17,18]. All the PCR products were purified and sequenced and the sequences were compared with the reference deposited in the GenBank nucleotide database.

\section{Conjugation experiments}


Luria-Bertani (LB) mating experiments were performed using sodium azide-resistant $E$. coli J53 as the recipient to determine whether the $16 \mathrm{~S}$ rRNA methylase genes or the carbapenemase genes are transferable.Transconjugants were selected on LB plates containing sodium azide (100 mg/L) plus gentamicin (30 mg/L) or imipenem (4 mg/L), and finally confirmed by PCR and pulsed field gel electrophoresis (PFGE)[19]. We also performed PCR to confirm whether the transconjugants also contained the other antibiotic-resistance genes that were found in the donor strains.

\section{PFGE and MLST}

All the CR-hvKP isolates were subjected to PFGE after digestion with $\mathrm{Xbal}$, as previously described ${ }^{20}$. The cluster cutoff line at $80 \%$ similarity was used to analyze genetic relatedness. Seven conserved house keeping genes ( $g a p A$, infB, $m d h$, pgi, phoE, rpoB, tonB) were used to perform MLST

(http://www.pasteur.fr/recherche/genopole/ PF8/mlst/Klebsiellapneumoniae.html).

\section{Results}

\section{The clinical characteristics and the association of antibiotic susceptibilities with 16S rRNA methylase genes in CR-hvKP isolates}

Overall, 39 patients had proven or suspected acquisition of CP-hvKP, more than half of all patients (64.1\%) had the carbapenems application empirically, but only 7 patients (17.9\%) had the aminoglycosides application. On the basis of the presence of 16S rRNA methylase genes in these CR-hvKP strains, all CR-hvKP strains were divided into two groups (16S rRNA methylase genes-positive strains and 16S rRNA methylase genes-negative strains). PCR analysis of $16 \mathrm{~S}$ rRNA methylase genes revealed 26 (66.7\%) CR-hvKP isolates had 16S rRNA methylase genes and 13 (33.3\%) CR-hvKP isolates had no any $16 \mathrm{~S}$ rRNA methylase genes. There was no significant difference in eight antimicrobial susceptibilities except aminoglycosides susceptibilities between two groups. Twenty-six 16S rRNA methylase genes-positive strains were all resistant to Amikacin,gentamicin and tobramycin. Thirteen $16 \mathrm{~S}$ rRNA methylase genes-negative strains were all susceptible to amikacin and gentamicin, but only three 16S rRNA methylase genes-negative strains were still susceptible to tobramycin. Almost all the CR-hvKP strains were reistant to carbapenems,beta lactam antibiotics and quinolones, only one $16 \mathrm{~S}$ rRNA methylase genes-negative strain was susceptible to levofloxacin. Fortunately there were four 16S rRNA methylase genes-negative strains and eight 16S rRNA methylase genes-positive strains susceptible to co-trimoxazole. All the CR-hvKP strains exhibited the hypervirulence that all the patients had high ICU admission rate (51.2\%) and high mortality (58.9\%) in 30 days in spite of $16 \mathrm{~S}$ rRNA methylase genes (Table 1) .

\section{Prevalence of 16S rRNA methylase genes and other antimicrobial resistance genes among CR-hvKP clinical strains}

As shown in Table 2, 66.7\% (26/39) of the CR-hvKP isolates were found to carry at least one $16 \mathrm{~S}$ rRNA methylase gene, with armA, rmtB being detected alone or in combination in 11,8 , and 7 strains, respectively. However, $r m t A, n p m A, r m t C$ and $r m t D$ were not detected in these strains. All the clinical isolates were found to carry at least one carbapenemase gene,with KPC-2 (79.5\%,31/39),NDM-1 (10.3\%,4/39), cocarrying KPC-2 and NDM-1 $(10.3 \%, 4 / 39)$. However, IMP, VIM, OXA-48 were not detected in these strains. A total of $89.7 \%(35 / 39)$ isolates carried $\mathrm{ESBL}$ genes, including $61.5 \%$ (24/39) blaSHV-1,71.8\% (28/39) blaTEM-1 and 89.7\% (35/39) blaCTX-M-14.All except four isolates (89.7\%,35/39) harbored PMQR genes,with qnrS $(82.1 \%, 32 / 39), \operatorname{aac}\left(6^{\prime}\right)-\operatorname{lb}-\operatorname{cr}(79.5 \%, 31 / 39), \operatorname{qnr} B(2.6 \%, 1 / 39)$.All the $16 \mathrm{~S}$ rRNA methylase genes-positive CR-hvKP strains were firstly found to cocarry carbapenemase genes, ESBL genes and PMQR genes simultaneously (Table 2).

\section{Capsular serotyping K1/K2 and Virulence genes in CR-hvKP clinical strains}

Capsular serotyping showed that of 39 CR-hvKP strains, both four isolates were identified as capsular genotypes K1 and K2. The 14 investigated virulence genes were detected in the K. pneumoniae isolates and are shown in Table 2. Various virulence-associated genes are responsible for determining the pathogenicity of the $39 \mathrm{~K}$. pneumoniae isolates tested, including $\operatorname{mag} A(10.3 \%, 4 / 39), \operatorname{rmp} A(87.2 \%, 34 / 39)$, terW(84.6\%, 33/39), silS(97.4\%, 38/39), iutA(92.3\%, 36/39), rmpA2(100\%, 39/39), kfuBC (10.3\%, 4/39), wcaG (10.3\%, 4/39), allS (10.3\%, 4/39), kpn(92.3\%, 36/39), entB(100\%, 39/39), ybtS (94.9\%, 37/39), repA(17.9\%, 7/39), and aerobactin $(23.1 \%, 9 / 39)$. The positive rates of magA, kfuBC, wcaG, allS, and aerobactin among $\mathrm{K} 1 / \mathrm{K} 2$ isolates were significantly higher than non-K1/K2 isolates $(\mathrm{p}<0.05)$.

\section{Molecular characteristics}

The PFGE-based fingerprints of the CR-hvKP isolates displayed three different clusters (named A-C) using a similarity cutoff value of $80 \%$ (Fig. 1), including cluster A $(31 / 39,79.5 \%)$,cluster B $(4 / 39,10.3 \%)$ and cluster C $(4 / 39,10.3 \%)$. The MLST analysis distinguished a total of four different STs. The most prevalent ST in CR-hvKP isolates was ST11 (31/39, 79.5\%), followed by ST23 (4/39, 10.3\%), ST65 (2/39, 5.1\%), and ST86 (2/39, 5.1\%). Notably, MLST and PFGE yielded similar results, with 31 cluster A isolates belonging to ST11, 4 cluster B isolates belonging to ST23, and both two cluster C isolates belonging to ST65 and ST86, respectively. The PFGE-based fingerprints of ST11 KPC-2 hvKP from different wards were almost the same and the 16S rRNA methylase genes positive rate in this kind of CR-hvKP strains was quite high (67.7\%). Our data revealed that ST11 KPC-2 hvKP strains were highly transmissible clonally and were extremely easy to cocarry the 16S rRNA methylase genes.

\section{Conjugation experiments}

In conjugation experiments, twenty-six 16S rRNA methylase genes positive strains were used as donors. The transconjugation were successful in 17 (65.4\%) CR-hvKP strains, including 9 armA-positive strains, 5 rmtB-positive strains, 3 armA-rmtB-positive strains. 16S rRNA methylase genes were found in 12 transconjugants by PCR and only $r m t B$ was found in 3 armA-rmtB-positive transconjugants, indicating that only rmtB was present on the 
transferable plasmid in these armA-rmtB-positive strains. Additionally, the analysis of the transconjugants revealed a common cotransmission of bla KPC-2 with the 16S rRNA methylase genes that both 16S rRNA methylase genes and KPC-2 were positive in 9 transconjugants.

\section{Discussion}

Over the past few decades, hvKP has globally emerged,causing invasive infections since the first clinical hvKP report in 1986[21]. Although initial isolates of hvKp were antimicrobial sensitive,clonal complexes of hypervirulent (hvKP) and multidrug-resistant (MDR) strains are non-overlapping[22].In this study we collected 39 CR-hvKP strains, including 31 ST11 CRKP carrying the pLVPK like plasmid ,4 ST23, 2 ST65 and 2 ST86 hvKP carrying the carbapenemase plasmid.It was consistant with the evolution hypothesis of MDR-hvKP occurring by two mechanisms[21]. The first and more frequent was via XDR cKp acquiring a modified hvKp virulence plasmid, and the converse was via hvKp strains gaining antimicrobial resistance genes by acquisition of resistance plasmids or by the insertion of resistance elements into hvKp's virulence plasmid[5,21]. To date,though such CR-hvKP strains have been described only in China; the prospect of CR-hvKp undergoing wider dissemination is concerning.

Because of the expensive cost, the application of tigecycline and polymyxin in clinical treatment were severely limited.Considering that CRKP isolates were generally susceptible to aminoglycosides, these drugs were widely used for treating CRKP infections[6] in spite of ototoxicity and nephrotoxicity. However, increased manifestations of antimicrobial resistance mediated by plasmids, especially 16S rRNA methylase, and carbapenemase in MDR K. pneumoniae, had been observed in China[23]. In this study the 16S rRNA methylase genes were highly prevalent in CR-hvKP isolates, The most frequently detected 16S rRNA methylase genes was armA, followed by rmtB and both two in all isolates. As described in a previous study[24], 16S rRNA methylase genes confered high-level aminoglycoside resistance including amikacin,gentamicin and tobramycin in CR-hvKP strains this study.Previous studies had identified an increasing association between carbapenemase production and aminoglycoside resistance[9,23,25]. Carbapenemase encoding genes could be cotransferred with 16S rRNA methylase genes on the same mobile genetic elements[23]. Furthermore, increasing numbers of hvKP isolates with carbapenemase have been reported from different parts of China[3,26,27].In the present study, a high percentage $(52.9 \%, 9 / 17)$ of $16 \mathrm{~S}$ rRNA methylase gene armA or $r m t B$ was cotransferred with carbapenemase encoas the most cding gene $K P C-2$ among these CR-hvKP. Even more disturbing was that KPC-2-type carbapenemase wommon genotype in China. Interestingly we firstly found that carbapenemase gene NDM-1 and 16S rRNA methylase gene armA both were simultaneously present in one CR-hvKP strain this study, but whether these two drug resistance gene existed in the same plasmid needed further study by Southern-blot.

As previous studies reported several gene clusters were associated with virulence in hvKP K1/K2 serotype strains[28,29], virulence genes including aerobact, repA, kfuBC and wcaG were highly clustered in the K1/K2 16S rRNA methylase genes-positive CR-hvKP strains compared to non-K1/K2 16S rRNA methylase genes-positive strains. Our data showed that ST11 was the most prevalent among 16S rRNA methylase genes-positive CR-hvKP isolates. Among 21 ST11 isolates, the first and second isolates were emerged in January 2018 in our hospital, whereas 14 isolates were identified during the period from April to July 2017. In August and September, four ST11 isolates were identified, and the last isolate was found in December. Our data indicated that there was an outbreak of ST11 16S rRNA methylase genes-positive CR-hvKP in our hospital in 2018.Control measures should be implemented to prevent further dissemination of such organisms in the hospital setting, because the ST11 carbapenem-resistant hypervirulent $\mathrm{K}$ pneumoniae strains are simultaneously hypervirulent, multidrug resistant, and highly transmissible[4]. In addition, ST23, ST65 and ST86 were also found to be associated with 16S rRNA methylase genes-positive CR-hvKP.

Several potential limitations and caveats of this study are noteworthy, including its retrospective nature and a relatively small study population. In this study, 39 isolates used in the analysis were unable to clearly elucidate the 16S rRNA methylase genes-positive CR-hvKP epidemiology.Therefore, there may be selection bias, which limits the general application of study results to other areas.

\section{Conclusions}

In conclusion, 16S rRNA methylase genes are highly prevalent in CR-hvKP clinical isolates in our hospital, and 16S rRNA methylase genes-positve CRhvKP strains especially for ST11 will be difficult to eliminate and control because of both horizontal transfer and clonal spread.Furthermore, the $16 \mathrm{~S}$ rRNA methylase genes could be cotransferred with blaKPC, suggesting that CR-hvKP could acquire transferable resistance elements as independent events from an external source, of which we should keep alert

\section{Abbreviations}

CR-hvKP:carbapenem-resistant hypervirulent Klebsiella pneumoniae

CRKP:carbapenem-resistant Klebsiella pneumoniae hvKP:hypervirulent Klebsiella pneumoniae

PMQR: plasmid-mediated quinolone resistance MLST:multilocus sequence typing

PFGE:Pulsed-field gel electrophoresis

\section{Declarations}

\section{Ethics approval and consent to participate}


The study has been evaluated by the Ethics Committee of the First Affiliated Hospital of Nanchang University. Patients involved in the study were anonymized, no informed consent was acquired because of the retrospective study.

\section{Consent for publication}

Not applicable

\section{Availability of data and material}

The data that support the findings of this study are available from the corresponding author upon reasonable request

\section{Competing interests}

The author reports no conflicts of interest in this work.

\section{Funding}

Financial support was provided by the National Natural Science Foundation of China (81860368).

\section{Author contributions}

FLD and DL performed the laboratory measurements. YL and WJL made substantial contributions to conception and design. WZ and YL revised the manuscript critically for important intellectual content. DL and DDW participated in experimental design and data analysis. WJL drafted the manuscript. All authors read and approved the final manuscript.

\section{Acknowledgments}

Not applicable.

\section{References}

1. Paczosa MK, Mecsas J: Klebsiella pneumoniae: Going on the Offense with a Strong Defense. Microbiol Mol Biol Rev 2016, 80:629-661.

2. Dong N, Liu L, Zhang R, Chen K, Xie M, EWC C, Chen S: An IncR Plasmid Harbored by a Hypervirulent Carbapenem-Resistant Klebsiella pneumoniae Strain Possesses Five Tandem Repeats of the blaKPC-2::NTEKPC-Id Fragment. Antimicrob Agents Chemother 2019, 63:

3. Liu Y, Long D, Xiang TX, et al. Whole genome assembly and functional portrait of hypervirulent extensively drug-resistant NDM-1 and KPC-2 coproducing Klebsiella pneumoniae of capsular serotype K2 and ST86. J Antimicrob Chemother. 2019.

4. Gu D, Dong N, Zheng Z, et al. A fatal outbreak of ST11 carbapenem-resistant hypervirulent Klebsiella pneumoniae in a Chinese hospital: a molecular epidemiological study. Lancet Infect Dis. 2018. 18(1): 37-46.

5. Wyres KL, Wick RR, Judd LM, et al. Distinct evolutionary dynamics of horizontal gene transfer in drug resistant and virulent clones of Klebsiella pneumoniae. PLoS Genet. 2019. 15(4): e1008114.

6. Satlin MJ, Kubin CJ, Blumenthal JS, et al. Comparative effectiveness of aminoglycosides, polymyxin B, and tigecycline for clearance of carbapenem-resistant Klebsiella pneumoniae from urine. Antimicrob Agents Chemother. 2011. 55(12): 5893-9.

7. Petrosillo N, Giannella M, Lewis R, Viale P. Treatment of carbapenem-resistant Klebsiella pneumoniae: the state of the art. Expert Rev Anti Infect Ther. 2013. 11(2): 159-77.

8. Doi Y, Wachino Jl, Arakawa Y. Aminoglycoside Resistance: The Emergence of Acquired 16S Ribosomal RNA Methyltransferases. Infect Dis Clin North Am. 2016. 30(2): 523-537.

9. Tada T, Tsuchiya M, Shimada K, et al. Dissemination of Carbapenem-resistant Klebsiella pneumoniae clinical isolates with various combinations of Carbapenemases (KPC-2, NDM-1, NDM-4, and OXA-48) and 16S rRNA Methylases (RmtB and RmtC) in Vietnam. BMC Infect Dis. 2017. $17(1)$ : 467.

10. Tada T, Miyoshi-Akiyama T, Dahal RK, et al. Dissemination of multidrug-resistant Klebsiella pneumoniae clinical isolates with various combinations of carbapenemases (NDM-1 and OXA-72) and 16S rRNA methylases (ArmA, RmtC and RmtF) in Nepal. Int J Antimicrob Agents. 2013. 42(4): 372-4.

11. Xu M, Fu Y, Fang Y, et al. High prevalence of KPC-2-producing hypervirulent Klebsiella pneumoniae causing meningitis in Eastern China. Infect Drug Resist. 2019. 12: 641-653.

12. Humphries RM, Ambler J, Mitchell SL, et al. CLSI Methods Development and Standardization Working Group Best Practices for Evaluation of Antimicrobial Susceptibility Tests. J Clin Microbiol. 2018. 56(4).

13. Li P, Liu D, Zhang X, et al. Characterization of Plasmid-Mediated Quinolone Resistance in Gram-Negative Bacterial Strains from Animals and Humans in China. Microb Drug Resist. 2019

14. Kazemian H, Heidari H, Ghanavati R, et al. Phenotypic and Genotypic characterization of ESBLs, AmpC and Carbapenemase-Producing Klebsiella pneumoniae and Escherichia coli Isolates. Med Princ Pract. 2019.

15. Liu Y, Wan LG, Deng Q, Cao XW, Yu Y, Xu QF. First description of NDM-1-, KPC-2-, VIM-2- and IMP-4-producing Klebsiella pneumoniae strains in a single Chinese teaching hospital. Epidemiol Infect. 2015. 143(2): 376-84. 
16. Yeganeh SF, Mohammadzadeh-AsI Y, Ghotaslou R. High-Level Resistance to Aminoglycosides due to 16S rRNA Methylation in Enterobacteriaceae Isolates. Microb Drug Resist. 2019.

17. Turton JF, Perry C, Elgohari S, Hampton CV. PCR characterization and typing of Klebsiella pneumoniae using capsular type-specific, variable number tandem repeat and virulence gene targets. J Med Microbiol. 2010. 59(Pt 5): 541-7.

18. Candan ED, Aksöz N. Klebsiella pneumoniae: characteristics of carbapenem resistance and virulence factors. Acta Biochim Pol. 2015. 62(4): 867-74.

19. Liu Y, Du FL, Xiang TX, et al. High Prevalence of Plasmid-Mediated Quinolone Resistance Determinants Among Serotype K1 Hypervirulent Klebsiella pneumoniae Isolates in China. Microb Drug Resist. 2019. 25(5): 681-689.

20. Wang Z, Li M, Shen X, et al. Outbreak of blaNDM-5-Harboring Klebsiella pneumoniae ST290 in a Tertiary Hospital in China. Microb Drug Resist. 2019

21. Russo TA, Marr CM. Hypervirulent Klebsiella pneumoniae. Clin Microbiol Rev. 2019. 32(3).

22. Hennequin C, Robin F. Correlation between antimicrobial resistance and virulence in Klebsiella pneumoniae. Eur J Clin Microbiol Infect Dis. 2016. 35(3): 333-41.

23. Wei DD, Wan LG, Yu Y, et al. Characterization of extended-spectrum beta-lactamase, carbapenemase, and plasmid quinolone determinants in Klebsiella pneumoniae isolates carrying distinct types of 16S rRNA methylase genes, and their association with mobile genetic elements. Microb Drug Resist. 2015. 21(2): 186-93.

24. Yan JJ, Wu JJ, Ko WC, et al. Plasmid-mediated 16S rRNA methylases conferring high-level aminoglycoside resistance in Escherichia coli and Klebsiella pneumoniae isolates from two Taiwanese hospitals. J Antimicrob Chemother. 2004. 54(6): 1007-12.

25. Taylor E, Sriskandan S, Woodford N, Hopkins KL. High prevalence of 16 S rRNA methyltransferases among carbapenemase-producing Enterobacteriaceae in the UK and Ireland. Int J Antimicrob Agents. 2018. 52(2): 278-282.

26. Yao B, Xiao X, Wang F, Zhou L, Zhang X, Zhang J. Clinical and molecular characteristics of multi-clone carbapenem-resistant hypervirulent (hypermucoviscous) Klebsiella pneumoniae isolates in a tertiary hospital in Beijing, China. Int J Infect Dis. 2015. 37: 107-12.

27. Liu BT, Su WQ. Whole genome sequencing of NDM-1-producing serotype K1 ST23 hypervirulent Klebsiella pneumoniae in China. J Med Microbiol. 2019. 68(6): 866-873.

28. Turton JF, Payne Z, Coward A, et al. Virulence genes in isolates of Klebsiella pneumoniae from the UK during 2016, including among carbapenemase gene-positive hypervirulent K1-ST23 and 'non-hypervirulent' types ST147, ST15 and ST383. J Med Microbiol. 2018. 67(1): 118-128.

29. Yu WL, Ko WC, Cheng KC, Lee CC, Lai CC, Chuang YC. Comparison of prevalence of virulence factors for Klebsiella pneumoniae liver abscesses between isolates with capsular K1/K2 and non-K1/K2 serotypes. Diagn Microbiol Infect Dis. 2008. 62(1): 1-6.

\section{Tables}

Table 1/The clinical characteristics and the association of antibiotic susceptibilities with 16S rRNA methylase genes in CR-hvKP isolates 


\begin{tabular}{|c|c|c|c|}
\hline & \multicolumn{2}{|c|}{ 16S rRNA methylase genes } & \multirow{2}{*}{ P-value } \\
\hline & Positive $(n=26)$ & $\overline{\text { Negative }(n=13)}$ & \\
\hline \multicolumn{4}{|l|}{ Clinical characteristics } \\
\hline Age(year>65) & 7 & 5 & 0.713 \\
\hline Sex(male) & 20 & 11 & 0.888 \\
\hline Brain injury & 16 & 10 & 0.548 \\
\hline Diabetes & 6 & 1 & 0.388 \\
\hline Hypertension & 10 & 2 & 0.270 \\
\hline Pulmonary infection & 12 & 5 & 0.648 \\
\hline Carbapenems application & 17 & 8 & 0.813 \\
\hline Aminoglycosides application & 5 & 2 & 0.571 \\
\hline Quinolones application & 4 & 1 & 0.468 \\
\hline Any ICU admission & 13 & 7 & 0.821 \\
\hline Mortality in 30 days & 16 & 7 & 0.645 \\
\hline \multicolumn{4}{|l|}{ Antimicrobal susceptibility } \\
\hline Levofloxacin & 0 & 1 & NA \\
\hline Tobramycin & 0 & 3 & 0.037 \\
\hline Gentamincin & 0 & 11 & $<0.001$ \\
\hline Piperacillin tazobactam & 0 & 0 & NA \\
\hline Trimethoprim & 8 & 4 & 0.637 \\
\hline AmiKacin & 0 & 13 & $<0.001$ \\
\hline Ceftazidime & 0 & 0 & NA \\
\hline Aztreonam & 0 & 0 & NA \\
\hline Imipenem/Meropenem & 0 & 0 & NA \\
\hline Cefazolin & 0 & 0 & NA \\
\hline Ceftriaxone & 0 & 0 & NA \\
\hline
\end{tabular}

Statistically significant correlations $(p<0.05)$ are shown in bold font. 


\begin{tabular}{cccc}
\hline Specimen & Virulence genes & $\begin{array}{c}\text { 16SrRNA carbapenemase genes } \\
\text { methylase } \\
\text { gene }\end{array}$ & Other resistant genes
\end{tabular}

Table 2. Main

Features of all

: CR-hvKP isolates

and the drug

resistance

genes results

of 16SrRNA

methylase gene-

positive CR-

کP transconjugants

\begin{tabular}{|c|c|c|c|c|c|}
\hline Kp1 & Sputum & $\begin{array}{l}\text { rmpA, terW, silS, iutA, rmpA2 } \\
\text { kpn, entB, ytbs, kpn, entB, ytbs }\end{array}$ & - & $K P C-2$ & $S H V-1, C T X-M-14, q n r S, a C C\left(6^{\prime}\right)-I b-c r$ \\
\hline Kp2 & Urine & $\begin{array}{l}r m p A, t e r W, \text { sils, iutA, rmpA2 } \\
\text { kpn, entB, ytbs, kpn, entB, ytbs }\end{array}$ & - & $K P C-2$ & $C T X-M-14, q n r S, a c c\left(6^{\prime}\right)-I b-c r$ \\
\hline Kp3 & Blood & $\begin{array}{l}r m p A, \text { terW, silS,iutA,rmpA2, } \\
\text { kpn,entB,ytbs, aerobact }\end{array}$ & - & $K P C-2$ & TEM-1,CTX-M-14, qnrS, acc $\left(6^{\prime}\right)-I b-c r$ \\
\hline Kp4 & Blood & $\begin{array}{l}r m p A, \text { terW, silS, iutA, rmpA2 } \\
\text { kpn,entB,ytbs }\end{array}$ & - & $K P C-2$ & $\begin{array}{c}\text { SHV-1, TEM-1,CTX-M-14, qnrS, } \\
\text { acc }\left(6^{\prime}\right)-I b-c r\end{array}$ \\
\hline Kp5 & Blood & $\begin{array}{c}\text { rmpA, terW, silS, iutA,rmpA2 } \\
\text { kpn,entB,ytbs }\end{array}$ & - & $K P C-2$ & $\begin{array}{c}S H V-1, T E M-1, C T X-M-14, q n r S \\
\operatorname{acc}\left(6^{\prime}\right)-I b-c r\end{array}$ \\
\hline Kp6 & Blood & $\begin{array}{l}\text { rmpA, terW, silS, iutA, rmpA2 } \\
\text { kpn, entB,ytbs }\end{array}$ & - & $K P C-2$ & $\begin{array}{c}T E M-1, C T X-M-14, q n r S \\
\text { acc }\left(6^{\prime}\right)-I b-C r\end{array}$ \\
\hline Kp7 & Blood & $\begin{array}{c}r m p A, \text { terW,silS,iutA, rmpA2 } \\
\text { kpn,entB,ytbs }\end{array}$ & - & $K P C-2$ & $\begin{array}{c}T E M-1, C T X-M-14, q n r S \\
\text { acc }\left(6^{\prime}\right)-I b-C r\end{array}$ \\
\hline Kp8 & Blood & $\begin{array}{c}\text { rmpA, terW, silS,iutA, rmpA2 } \\
\text { kpn, entB,ytbs }\end{array}$ & - & $K P C-2$ & $\begin{array}{c}S H V-1, T E M-1, C T X-M-14, q n r S, \\
\operatorname{acc}\left(6^{\prime}\right)-I b-c r\end{array}$ \\
\hline Kp9 & Blood & $\begin{array}{l}\text { rmpA,terW, silS,iutA, rmpA2 } \\
\text { kpn,entB,ytbs }\end{array}$ & - & $K P C-2$ & $\begin{array}{c}S H V-1, T E M-1, C T X-M-14, q n r S, \\
\operatorname{acc}\left(6^{\prime}\right)-I b-c r\end{array}$ \\
\hline Kp10 & Blood & $\begin{array}{l}r m p A, \text { terW, silS, iutA, } r m p A 2 \\
\text { kpn,entB,ytbs }\end{array}$ & - & $K P C-2$ & $\begin{array}{c}S H V-1, T E M-1, C T X-M-14, q n r S \\
\text { a cC }\left(6^{\prime}\right)-I b-c r\end{array}$ \\
\hline Kp11 & Sputum & $\begin{array}{c}\text { rmpA, terW, silS,iutA,rmpA2 } \\
\text { kpn,entB,ytbs }\end{array}$ & $\operatorname{arm} A$ & $K P C-2$ & $\begin{array}{c}S H V-1, T E M-1, C T X-M-14, q n r S, \\
\text { acc }\left(6^{\prime}\right)-I b-c r\end{array}$ \\
\hline T-KP11 & - & - & $\operatorname{arm} A$ & $K P C-2$ & $T E M-1$ \\
\hline Kp12 & Sputum & $\begin{array}{l}r m p A, \text {,sils,iutA,rmpA2 } \\
\text { kpn,entB,ytbs }\end{array}$ & $r m t B$ & $N D M-1 \square K P C-2$ & $S H V-1, T E M-1, C T X-M-14, q n r S$ \\
\hline T-KP12 & - & - & - & - & - \\
\hline Kp13 & Sputum & $\begin{array}{l}\text { rmpA, terW,silS,iutA, rmpA2 } \\
\text { kpn,entB,ytbs }\end{array}$ & $r m t B$ & $K P C-2$ & $\begin{array}{c}S H V-1, T E M-1, C T X-M-14 \\
\operatorname{acc}\left(6^{\prime}\right)-I b-c r\end{array}$ \\
\hline T-KP13 & - & - & $r m t B$ & $K P C-2$ & TEM-1, acc $\left(6^{\prime}\right)-I b-c r$ \\
\hline Kp14 & Sputum & $\begin{array}{l}\text { rmpA, terW, silS,iutA, rmpA2 } \\
\text { kpn,entB,ytbs }\end{array}$ & $\operatorname{arm} A \| r m t B$ & $K P C-2$ & $\begin{array}{c}S H V-1, T E M-1, C T X-M-14, q n r S \\
\text { acc }\left(6^{\prime}\right)-I b-c r\end{array}$ \\
\hline T-KP14 & - & - & $r m t B$ & $K P C-2$ & TEM-1,qnrS, \\
\hline Kp15 & Sputum & $\begin{array}{l}r m p A, \text { sils,iutA, rmpA2 } \\
\text { kpn,entB,ytbs, repA }\end{array}$ & $\operatorname{arm} A$ & $N D M-1 \square K P C-2$ & $\begin{array}{c}S H V-1, T E M-1, C T X-M-14, q n r S, \\
\operatorname{acc}\left(6^{\prime}\right)-I b-c r\end{array}$ \\
\hline T-KP15 & - & - & $\operatorname{arm} A$ & - & $T E M-1, a c c\left(6^{\prime}\right)-I b-C r$ \\
\hline Kp16 & Balf & $\begin{array}{l}r m p A, \text {,sils,iutA, rmp } A 2 \\
\text { kpn,entB,ytbs }\end{array}$ & $\operatorname{arm} A$ & $N D M-1 \square K P C-2$ & $\begin{array}{c}S H V-1, T E M-1, C T X-M-14, q n r S \\
\text { a cC }\left(6^{\prime}\right)-I b-C r\end{array}$ \\
\hline T-KP16 & - & - & $\operatorname{arm} A$ & - & \\
\hline
\end{tabular}

Continued

TEM-1, a cC (6')-Ib-cr

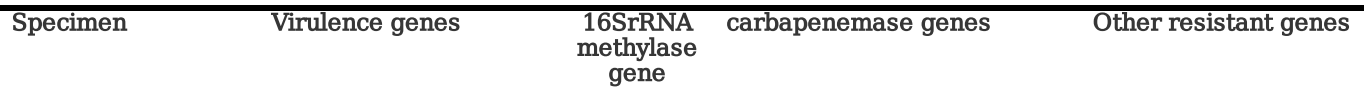

able 2. Continued 


\begin{tabular}{|c|c|c|c|c|c|}
\hline Kp17 & Sputum & $\begin{array}{c}\text { rmpA, terW, silS, iutA, rmpA2 } \\
\text { kpn, entB, ytbs }\end{array}$ & $\operatorname{armA}$ & $K P C-2$ & $\begin{array}{c}S H V-1, C T X-M-14, q n r S \\
a c C\left(6^{\prime}\right)-I b-c r\end{array}$ \\
\hline T-KP17 & - & - & - & - & - \\
\hline Kp18 & Blood & $\begin{array}{l}\text { rmpA, terW, silS, iutA, rmpA2 } \\
\text { kpn, entB,ytbs }\end{array}$ & $\operatorname{arm} A$ & $K P C-2$ & TEM-1,CTX-M-14, qnrS, acC $\left(6^{\prime}\right)-I b-c r$ \\
\hline T-KP18 & - & - & - & $K P C-2$ & $T E M-1, a c c\left(6^{\prime}\right)-I b-c r$ \\
\hline Kp19 & Blood & $\begin{array}{l}r m p A, \text { terW, silS,iutA, rmpA2 } \\
\text { kpn,entB,ytbs, repA, a erobact }\end{array}$ & $\operatorname{arm} A$ & $K P C-2$ & $\begin{array}{c}S H V-1, T E M-1, C T X-M-14, q n r S \\
\operatorname{acc}\left(6^{\prime}\right)-I b-c r\end{array}$ \\
\hline T-KP19 & - & - & $\operatorname{arm} A$ & - & CTX-M-14 \\
\hline Kp20 & Sputum & $\begin{array}{c}\text { terW, sils,iutA,rmpA2,kpn, } \\
\text { entB,ytbs }\end{array}$ & $\operatorname{arm} A$ & $K P C-2$ & $T E M-1, C T X-M-14, q n r S, a c c\left(\sigma^{\prime}\right)-I b-c r$ \\
\hline T-KP20 & - & - & - & $K P C-2$ & $T E M-1, a c c\left(6^{\prime}\right)-I b-c r$ \\
\hline Kp21 & Sputum & $\begin{array}{l}\text { terW, silS,iutA,rmpA2 } \\
\text { kpn,entB,ytbs, a erobact }\end{array}$ & $\operatorname{arm} A$ & $K P C-2$ & $\begin{array}{c}\text { TEM-1,CTX-M-14, qnrB, qnrS, } \\
\text { acc }\left(6^{\prime}\right)-I b-c r\end{array}$ \\
\hline T-KP21 & - & - & $\operatorname{arm} A$ & $K P C-2$ & $C T X-M-14$ \\
\hline Kp22 & Blood & $\begin{array}{c}r m p A, \text { sils,iutA,rmpA2, } k p n, \\
\text { entB,ytbs }\end{array}$ & $\operatorname{arm} A$ & $K P C-2$ & $\begin{array}{c}S H V-1, T E M-1, C T X-M-14, q n r S, \\
\operatorname{acc}\left(6^{\prime}\right)-I b-C r\end{array}$ \\
\hline T-KP22 & - & - & - & - & - \\
\hline Kp23 & Blood & $\begin{array}{c}r m p A, \text { sils,iutA,rmpA2, kpn, } \\
\text { entB,ytbs }\end{array}$ & $r m t B$ & $K P C-2$ & $\begin{array}{c}S H V-1, T E M-1, C T X-M-14, q n r S \\
\operatorname{acc}\left(6^{\prime}\right)-I b-c r\end{array}$ \\
\hline T-KP23 & - & - & $r m t B$ & $K P C-2$ & TEM-1,qnrS \\
\hline Kp24 & Blood & $\begin{array}{c}r m p A, \text { sils,iutA,rmpA2,kpn, } \\
\text { entB,ytbs }\end{array}$ & $r m t B$ & $K P C-2$ & $S H V-1, T E M-1, C T X-M-14, q n r S$ \\
\hline T-KP24 & - & - & $r m t B$ & $K P C-2$ & TEM-1,qnrS \\
\hline Kp25 & Blood & $\begin{array}{c}\text { rmpA, terW, silS, iutA, rmpA2 } \\
\text { kpn, entB,ytbs }\end{array}$ & $r m t B$ & $K P C-2$ & $\begin{array}{c}S H V-1, T E M-1, C T X-M-14, q n r S, \\
\operatorname{acc}\left(6^{\prime}\right)-I b-C r\end{array}$ \\
\hline T-KP25 & - & - & - & $K P C-2$ & $T E M-1, a c c\left(6^{\prime}\right)-I b-c r$ \\
\hline Kp26 & Blood & $\begin{array}{c}\text { rmpA, terW, sils,iutA, rmpA2 } \\
\text { kpn,entB,ytbs }\end{array}$ & $r m t B$ & $K P C-2$ & TEM-1,CTX-M-14, qnrS, acc $\left(6^{\prime}\right)-I b-c r$ \\
\hline T-KP26 & - & - & $r m t B$ & $K P C-2$ & TEM-1,qnrS \\
\hline Kp27 & Blood & $\begin{array}{c}r m p A, \text { terW, silS, iutA, rmpA2 } \\
\text { kpn, entB,ytbs }\end{array}$ & armA』rmtB & $K P C-2$ & TEM-1,CTX-M-14, qnrS, acc( $\left.6^{\prime}\right)-I b-c r$ \\
\hline
\end{tabular}

T-KP27

Continued

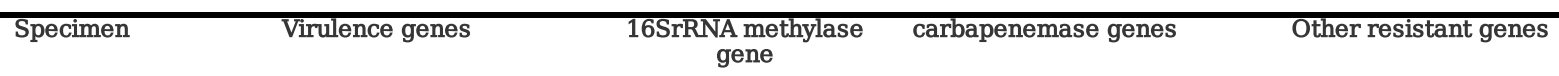

able 2. Continued

Isolates

Kp28

Blood

rmpA, terW, sils, iutA, rmpA2

kpn,entB, ytbs

armA $\triangle r m t B$

KPC-2

SHV-1,TEM-1,CTX-M-14, qnrS,

T-KP28

Kp29

Sputum

terW, silS, rmpA2
kpn, entB,ytbs, repA

$\operatorname{arm} A \not r m t B$

KPC-2 acc $\left(6^{\prime}\right)-I b-c r$

\section{T-KP29}

Kp30

Sputum

terW, silS,rmpA2 kpn, entB,ytbs, repA

armA $\ r m t B$

KPC-2

$K P C-2$

$r m t B$

SHV-1,TEM-1,CTX-M-14, qnrS, a cc $\left(6^{\prime}\right)-I b-c r$

Page 9/11 


\begin{tabular}{|c|c|c|c|c|c|}
\hline Kp31 & Sputum & $\begin{array}{l}r m p A, \text { terW, silS, rmpA2 } \\
\text { kpn,entB,ytbs,repA }\end{array}$ & $\operatorname{arm} A$ & $K P C-2$ & $\begin{array}{c}S H V-1, T E M-1, C T X-M-14, q n r B, \\
q n r S, \operatorname{acc}\left(6^{\prime}\right)-I b-c r\end{array}$ \\
\hline T-KP31 & - & - & - & $K P C-2$ & $C T X-M-14, q n r S$ \\
\hline Kp32 & Blood & $\begin{array}{l}\text { magA,rmpA, terW, silS, iutA, rmpA2, } \\
\text { kfuBC, wcaG, allS, entB, ytbs, aerobact }\end{array}$ & - & $N D M-1$ & qnrS,acc(6')-Ib-cr \\
\hline Kp33 & Pus & $\begin{array}{l}\operatorname{magA}, \text { rmpA,terW, silS, iutA, rmpA2, } \\
\text { kfuBC, wcaG,allS, entB,ytbs, aerobact }\end{array}$ & - & $N D M-1$ & - \\
\hline Kp34 & Blood & $\begin{array}{l}\text { magA,rmpA, terW, silS, iutA, rmpA2, } \\
\text { kfuBC, wcaG , allS, entB, ytbs, aerobact }\end{array}$ & - & $K P C-2$ & - \\
\hline Kp35 & Sputum & $\begin{array}{c}\text { magA,rmpA, terW, silS, iutA, rmpA2, } \\
\text { kfuBC,wcaG,allS, kpn, entB, ytbs, aerobact }\end{array}$ & $\operatorname{arm} A$ & $N D M-1$ & $T E M-1, a c c\left(6^{\prime}\right)-I b-C r$ \\
\hline T-KP35 & - & - & - & $N D M-1$ & - \\
\hline Kp36 & Blood & $\begin{array}{l}\text { rmpA,terW, silS,iutA, rmpA2, } \\
\text { kpn,entB,ytbs }\end{array}$ & $\operatorname{arm} A / r m t B$ & $K P C-2$ & $\begin{array}{c}S H V-1, T E M-1, C T X-M-14, q n r S, \\
\operatorname{acc}\left(6^{\prime}\right)-I b-C r\end{array}$ \\
\hline T-KP36 & - & - & $r m t B$ & $K P C-2$ & $T E M-1, a c c\left(6^{\prime}\right)-I b-C r$ \\
\hline Kp37 & Blood & terW,iutA,rmpA2,kpn,entB,ytbs,repA & $\operatorname{arm} A \rrbracket r m t B$ & $K P C-2$ & SHV-1,TEM-1,CTX-M-14,qnrB \\
\hline T-KP37 & - & - & - & - & - \\
\hline Kp38 & Sputum & $\begin{array}{l}r m p A, \text { terW, sils,iutA,rmpA2 } \\
\text { kpn, entB, repA, aerobact }\end{array}$ & $r m t B$ & $N D M-1 \square K P C-2$ & $\begin{array}{c}T E M-1, C T X-M-14, q n r S \\
\operatorname{acC}\left(\sigma^{\prime}\right)-I b-C r\end{array}$ \\
\hline T-KP38 & - & - & - & - & - \\
\hline Kp39 & Balf & $\begin{array}{c}\text { rmpA,terW, silS,iutA,rmpA2 } \\
\text { kpn,entB,aerobact }\end{array}$ & $r m t B$ & $N D M-1$ & $\begin{array}{c}\text { TEM-1, } C T X-M-14, q n r S \\
\text { acc }\left(6^{\prime}\right)-I b-C r\end{array}$ \\
\hline T-KP39 & - & - & & - & - \\
\hline J53 & - & - & - & - & - \\
\hline
\end{tabular}

\section{Figures}

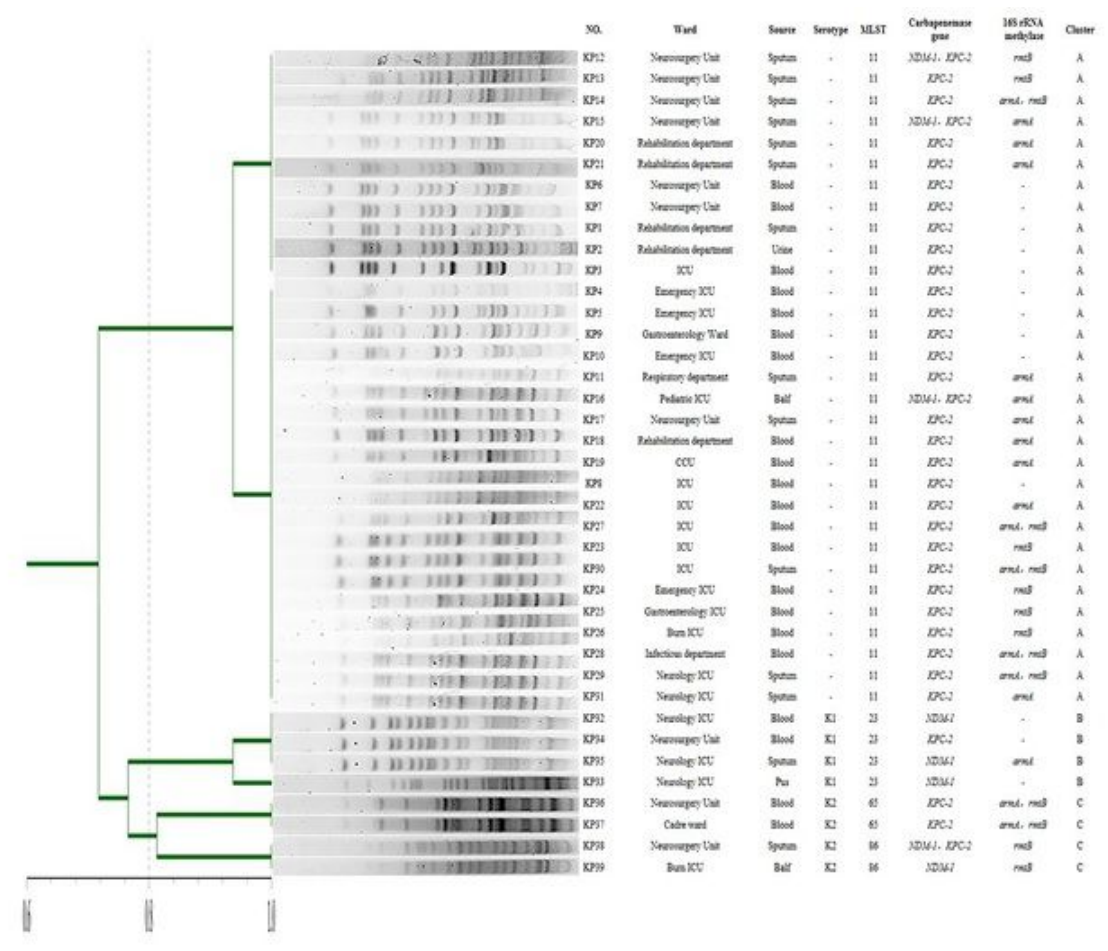

Figure 1

PFGE and MLST analysis of CR-hvKP isolates, the PFGE patterns have been organized according to a dendrogram of 39 CR-hvKP isolates based on MLST analysis. 


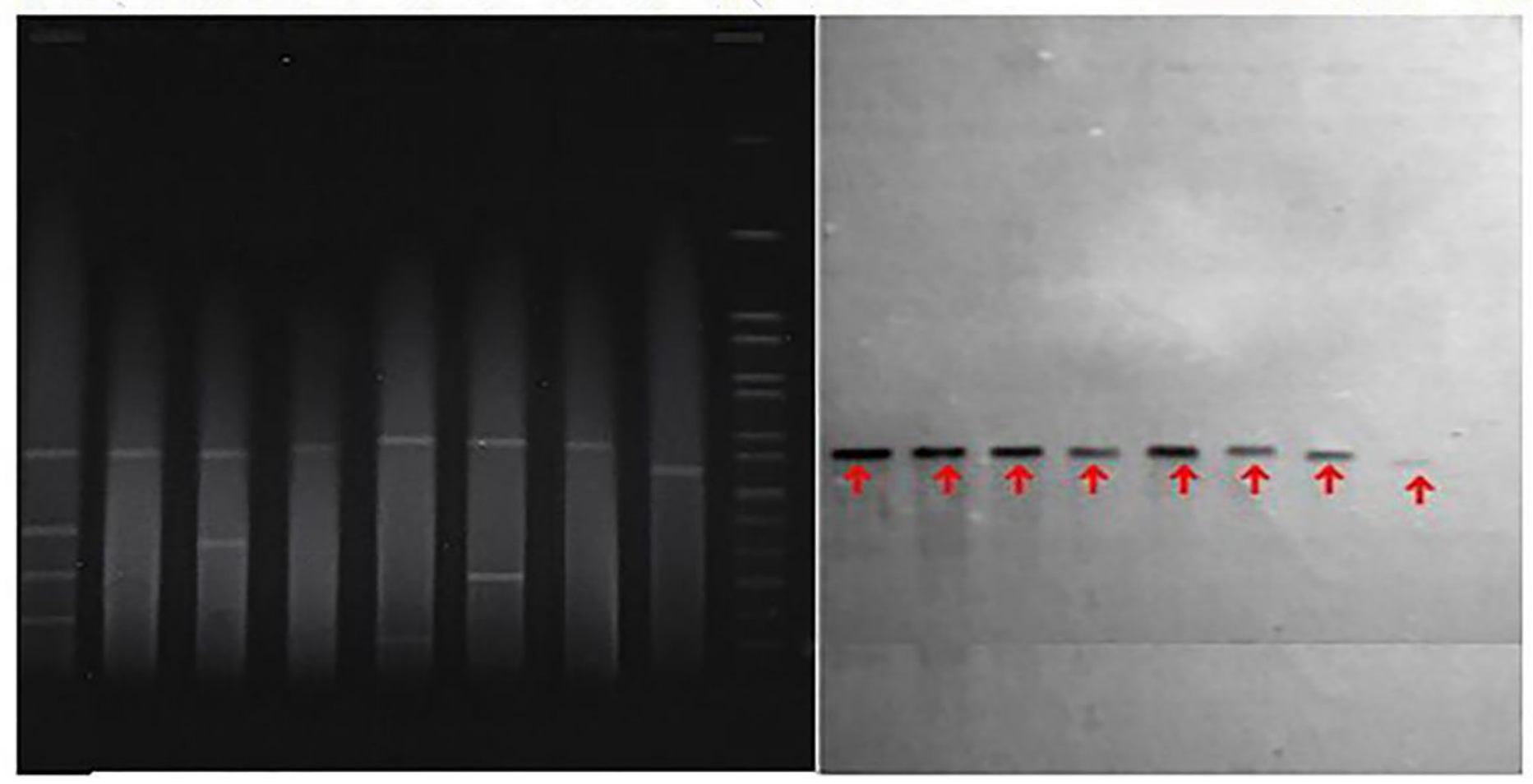

\section{Figure 2}

S1-PFGE and Southern hybridisation of a portion of CR-hvKP strains in this study A total of 39 nonduplicate CR-hvKP clinical isolates were collected from the First Affiliated Hospital of Nanchang University in the southeastern region of China.we select K.pneumoniae strains carrying the pLVPK-like virulence plasmid as hvKP strains by S1-PFGE and Southern hybridisation. The marker gene of the virulence plasmid rmpA2 was hybridised to confirm the presence of the pLVPK-like virulence plasmid. 